\title{
Editorial: Immunotherapy in Multiple Myeloma
}

\author{
Nicola Giuliani ${ }^{1,2 *}$ and Fabio Malavasi ${ }^{3}$ \\ ${ }^{1}$ Department of Medicine and Surgery, University of Parma, Parma, Italy, ${ }^{2}$ U.O. di Ematologia e Centro Trapianti Midollo \\ Osseo, Azienda Ospedaliero-Universitaria di Parma, Parma, Italy, ${ }^{3}$ Department of Medical Science, University of Turin and \\ "Fondazione Ricerca Molinette", Turin, Italy
}

Keywords: multiple myeloma, immunotherapy, monoclonal Ab, CD38, immune checkpoints

Editorial on the Research Topic

Immunotherapy in Multiple Myeloma

\section{HONORING THE PAST}

Multiple myeloma (MM) is a hematological malignancy characterized by a high tendency to relapse and to become drug resistant. In the past, melphalan was considered the standard of the treatment for MM patients (1). Following, the introduction of thalidomide and the proteasome inhibitor bortezomib leaded to a significant improvement of the survival of MM patients: however, none of them reached the cure of the disease. These drugs introduced the concept of the treatment of MM patients targeting not only the malignant clone but also the microenvironment (2). In addition, the introduction of lenalidomide, a thalidomide derivative, expanded this concept by focusing to the immune-microenvironment (2). More recently, the introduction of the monoclonal antibodies (mAbs) seem to change the paradigm of MM treatment, highlighting the possibility to cure MM patients by an immunotherapeutic approach (3).

Immunotherapy is part of a concept that goes back to the beginning of 1900. The "magic bullet" opened the way to the objective of having a tool able to selectively eliminate target cells and at the same to modulate the immune response in a beneficial way. It was necessary to wait for several decades and apparently for unrelated findings coming from different fields before having a picture able to frame the view outside a simple anecdotal hope. The objective was initially made possible by combining results from basic science and the availability of mAbs, a reagent made of a homogeneous population of immunoglobulins (Ig), the main difference from the conventional antisera. $\mathrm{mAb}$ is hence able to recognize only a single epitope on the molecular target.

The second key event derived from the identification of a vast number of soluble factors, which share the feature of transmitting signals in the context of similar or different cells (the interleukins). Using this new tools, Reinherz et al. generated a panel of mAbs specific for surface molecules located on the surface of human T lymphocytes (4). At the same time, Smith et al. made available IL-2, a cytokine which made possible to expand clones of normal T lymphocytes (5). Combining these approaches, Reinherz et al. (4) were able to define murine reagents specific for molecules present on all $\mathrm{T}$ lymphocytes, while other ones were limited to subsets of the same cells. Another set of mAbs recognized molecules only present in single lymphocytes (defined as idiotypic). These findings modified the simplistic dogma that mAbs were only able to bind the target antigen and to induce cell lysis. This lead to the definition of the concept of agonistic antibodies: the translational inference is that the engagement by a mAb of selected domain of a molecule may surrogate the effects induced by a natural ligand of the same receptor, even when the ligand was not known.

The definition of immune check points molecules lead to the preparation of panels of mAbs able to induce or brake immune responses, according to distinct medical needs. The concept of immune 
modulation was further completed by the identification of activator effector $\mathrm{T}$ and $\mathrm{B}$ cells, while other cell subsets produce effects going in opposite directions (Treg, Breg, and myeloid-derived suppressor cells are the most important). The considerations derived from the dissection of the main steps of immune cell defense were indirectly confirmed by studies conducted on the different strategies of immune escape of tumors. Indeed, it emerged that some tumors adopt escape or camouflage strategies, which implement genetic programs driving to metabolic reshaping, secretion of immunomodulatory cytokines, or generation of tolerogenic substances, among the others.

\section{STUDYING THE PRESENT}

At the moment, available to the medical community there is a panel of therapeutic antibodies, which significantly modified the fate of some diseases, especially neoplasies of hematological origin. There is a general agreement that the therapeutic effects are prevalently obtained by means of antibody-dependent cellular cytotoxicity (ADCC), antibody-dependent cell-mediated phagocytosis (ADCP), and complement-dependent cytotoxicity (CDC), and concurrently by the induction of signals on cell effectors (6). These effects may be enriched by other functions exerted by the target molecule, such as the ability to lead to generation of substrates able to induce immunomodulation. This is the case when a molecule belongs to the family of ectoenzymes, which now encompasses almost $5 \%$ of the entire surface molecule express by leukocyte (6). Other mAbs were generated against soluble molecules produced by both $\mathrm{MM}$ cells and the bone microenvironment including sclerostin able to block MMderived bone destruction and in turn MM progression.

In the treatment of MM, the Food and Drug Administration (FDA) approved daratumumab (DARA) and elotuzumab (Elo), two monoclonal IgG-k mAbs, specific for CD38 and SLAMF7 (signaling lymphocytic activation molecule F7), respectively. The approval was for the treatment of relapsed or refractory MM (RRMM) patients, in combination with lenalidomide and dexamethasone (7). CD38 is a transmembrane glycoprotein highly expressed on MM cells that acts as both a receptor and an ectoenzyme. It is also involved in the activation and proliferation of immune cells (Morandi et al.). SLAMF7 is a surface glycoprotein receptor expressed on plasma cells (PCs) and on natural killer (NK) cells that is implicated in adhesion to stromal cells and in the activation of NK cell effector function (8).

Both DARA and Elo share the feature to recruit the immune system to enhance cellular cytotoxicity directed against myeloma cells (9). However, Elo acts only through NK cells and its effects are improved in combination with immunomodulatory drugs (IMiDs) as lenalidomide. On the other hand, DARA and the newer isatuximab (Isa) shows a broad spectrum of activity, including $\mathrm{ADCC}, \mathrm{ADCP}, \mathrm{CDC}$, and possibly direct induction of apoptosis on MM cells. Further, they exhibit promising results even as a single-agent (9). Beyond mAbs against surface molecules, several agents targeting immune checkpoints (e.g., CTLA-4, LAG3, PD-1/PD-L1, ICOS) expressed on immune cells have also been recently developed as a therapeutic strategy to activate T-cell mediated anti-tumor immunity (10). Specifically, the PD-1/PD-L1 axis has emerged as a central immune checkpoint that controls anti-tumor immune responses and plays a critical role in the metabolic reprogramming of cancer cells within solid tumors. However, its role in $\mathrm{MM}$ progression remains to be clarified. Discordant results have been reported on $\mathrm{PD}-1 / \mathrm{PD}-\mathrm{L} 1$ expression in $\mathrm{MM}$ thus suggesting the need of a more precise definition of PD-1/PD-L1 distribution in the context of cells within the MM tumor microenvironment (Costa et al.). Interestingly, the expression of immunecheckpoint molecules by osteoclast has been recently underlined. However, single-agent studies on PD-1/PD-L1 inhibitors have not demonstrated significant responses in MM patients. On the other hand, other studies have demonstrated the ability of lenalidomide to enhance anti-MM immune activity mediated by PD-1/PD-L1 inhibition despite high grade of toxicity (Jelinek et al.).

The use of mAbs in therapy now led to the observation of antibody resistance, which may appear at different times. Different approaches were designed in order to answer this issue, which is of critical relevance in clinics. Hypothesis or observations explaining the effects may be referred to down modulation of the target molecule by the neoplastic cells. An alternative is represented by a re-distribution of the target molecule on selected surface domains (e.g., polar aggregation or capping). Polar aggregation tends to coalescence the CD38 molecule with ectoenzymes involved in the production of adenosine along with inhibitory complement receptors (CD46, CD55, and CD59) and PD-L1. The availability now of a second antibody with the same the same specificity but recognizing a different epitope may be proposed when the resistance to the first one is observed. This strategy is expected to bypass the resistance mechanisms and to exert new mechanisms of therapeutic action.

\section{TRYING TO DESIGN THE FUTURE}

The design of innovative strategies in $M M$ therapy is a difficult challenge, since the disease has been adopted as a model where different immunotherapeutic approaches are under evaluation. For these reasons, we would like to focus to some aspects, sometime not considered to design the future of the immunotherapy in MM. Most details and complete authoritative reviews may be found in the manuscripts of this Special Issue.

1) New target markers for mAb therapy. The efforts to identify specific markers exclusively identifying human myeloma cell surface has been quite disappointing. So far, a criteria adopted is quantitative (e.g., for anti-CD38) or based on clear receptorial features (e.g., for CS1 or SLAMF7). B cell maturation antigen (BCMA) was recently adopted in virtue of a quite restricted expression, along with APRIL, one of its ligand. All the potential targets for antibodymediated therapy are summarized in a recent and complete reviews $(11,12)$.

2) Cell-based therapies. Beside the mAb-mediated approaches alone or as drug carriers or bispecific $\mathrm{T}$ cell engager, the cellular approaches using genetically modified $\mathrm{T}$ lymphocytes (CAR-T) or NK cells are expanding exponentially and 
analyzed (11). Such approaches are reviewed in papers of the Special Issue.

3) Extension of NK cell life and activity. Strategies to extend the life and performance of NK cells is one of the hot areas in the field. Paiva group analyzed the gene profile obtained in NK cells exposed to Isa: among the up-modulated appeared CD137, an inducible molecule (also known as Tumor Necrosis Factor Receptor Super Family-9, TNFRSF-9) (13). For this molecule, there are available two different antibodies, used for clinical trials. Their use combined with Isa aimed at increasing the life span of NK cells produced unsatisfactory results, at least in the model adopted (13). However, new observations support the possibility of combinations between therapeutic anti-CD38 and anti-CD137. The disappointing results obtained in vivo with anti-CD137 mAbs (urelumab, a human IgG4, and utomilumab, a human IgG2) were likely attributable to negative effects mediated by their interactions with FcRs (14). Now a construct with an arm made of a recombinant trimetric form of the CD137 Ligand (TNFSF-9) associated to the different tumor-associated molecules leads to an in situ activation of CD8 cell co-stimulation, with production of IFN- $\gamma(15,16)$.

In order to generate potent antibodies against tumor cells and stimulating anti-tumor cell immunity, recently, trifunctional natural killer (NK) cell engagers (targeting NKp46 and CD16 on NK cells) and a tumor antigen on cancer cells have been developed. This approach produced in vitro more potent effects than the therapeutic antibodies used in clinics to target the same tumor antigens (17).

4) FcR engagement and effects induced by target ligation. No systematic analysis of this step was conducted on anti$\mathrm{CD} 38$ therapy to date. It is reasonable to expect that the differences in structure between DARA and Isa (one full human, the other one chimeric mAbs) may be reflected on the interactions with the IgG FcRs. Results obtained in vitro giving DARA-armed $\mathrm{FcR}^{+}$cells instead of soluble $\mathrm{mAb}$ is followed by a distinct membrane dynamics. Critical here are the effects induced by antibody ligation on the tumor target molecule. It is reported that this event may be followed by internalization of the target/antibody complex or externalization, followed by a release in the biological fluids in the form of microvesicles.

5) Combination therapies. Part of actual therapeutic potential of the different type of antibody approach may be improved by using immune modulators or combination with other mAbs (with similar or different specificities) or recombinant constructs. A limit on the use of reagents targeting one or two different molecules (surface targets or modulators of the immune response) comes from the recent evidence that the MM is characterized by a marked spatial genomic

\section{REFERENCES}

1. Cherry BM, Korde N, Kwok M, Roschewski M, Landgren O. Evolving therapeutic paradigms for multiple myeloma: back to the future. Leuk Lymphoma. (2013) 54:451-63. doi: 10.3109/10428194.2012.7 17277 heterogeneity, with an early phase with clonal sweeps followed by a regional evolution in advanced stages of the disease (18).

\section{AUTHOR CONTRIBUTIONS}

NG and FM equally contributed to write the manuscript and made a substantial, direct and intellectual contribution to the work, and approved it for publication.

\section{FUNDING}

This work was supported in part by a grant from the Associazione Italiana per la Ricerca sul Cancro IG2017 n. 20299 (NG) and the International Myeloma Foundation under 2018 Brian D. Novis Senior Research Grant (NG).

\section{ACKNOWLEDGMENTS}

We would like here to thank and remember Vito Pistoia (19492018). Vito was a brilliant witness of a lucky period of the italian renaissance, which blessed the italian medical research in the Seventies. After his M.D. graduation, he started a career in Genova, where a significant number of scientists with an international background were operating. The synergies among the groups of Benvenuto Pernis, Ruggero Ceppellini, Carlo Grossi, Manlio Ferrarini, Soldano Ferrone, and the Moretta group were the ground of his basic education in science. This was completed by a period in Alabama at the school of Max Cooper. B lymphocyte area became the first area of his interests.

Lately he became Director of the Oncology Lab at the Gaslini Institute (Genova, Italy), a further source of synergies with the groups of Lorenzo and Alessandro Moretta. His Lab became reference for studies of cancer microenvironment, seen as potential regulator (positively and negatively) of the immune responses.

From the above interests stemmed his interest on the analysis of the events taking place in the bone marrow and in human multiple myeloma, the focus of intense interest by basic and clinical scientists. His skills, experience in science, and personal enthusiasm was the starting point of the common effort behind this Special Issue.

His scientific personality was also enriched and completed by non-common personal qualities. His positive approach to life, his refined humor, and a smiling face (mirroring internal equilibrium and peace) were his master characteristics.

Unfortunately, Vito passed away before the completion of this Special Issue. For this, it is our honor and a pleasure for us to dedicate the Issue in his name and memory.

2. Kawano Y, Moschetta M, Manier S, Glavey S, Gorgun GT, Roccaro AM, et al. Targeting the bone marrow microenvironment in multiple myeloma. Immunol Rev. (2015) 263:160-72. doi: 10.1111/imr.12233

3. Franssen LE, Mutis T, Lokhorst HM, van de Donk NWCJ. Immunotherapy in myeloma: how far have we come? Ther Adv Hematol. (2019) 10:2040620718822660. doi: 10.1177/2040620718822660 
4. Reinherz EL, Parkman R, Rappeport J, Rosen FS, Schlossman SF. Aberrations of suppressor T cells in human graft-versus-host disease. N Engl J Med. (1979) 300:1061-8. doi: 10.1056/NEJM197905103001901

5. Smith KA, Lachman LB, Oppenheim JJ, Favata MF. The functional relationship of the interleukins. J Exp Med. (1980) 151:1551-6. doi: 10.1084/jem.151.6.1551

6. Malavasi F, Faini AC. Mechanism of action of a new anti-CD38 antibody: enhancing myeloma immunotherapy. Clin Cancer Res. (2019) 25:29468. doi: 10.1158/1078-0432.CCR-19-0260

7. Varga C, Maglio M, Ghobrial IM, Richardson PG. Current use of monoclonal antibodies in the treatment of multiple myeloma. Brit J Haematol. (2018) 181:447-59. doi: 10.1111/bjh.15121

8. Veillette A, Guo H. CS1, a SLAM family receptor involved in immune regulation, is a therapeutic target in multiple myeloma. Crit Rev Oncol Hematol. (2013) 88:168-77. doi: 10.1016/j.critrevonc.2013.04.003

9. Sherbenou DW, Mark TM, Forsberg P. Monoclonal antibodies in multiple myeloma: a new wave of the future. Clin Lymphoma Myeloma Leuk. (2017) 17:545-54. doi: 10.1016/j.clml.2017.06.030

10. Ok CY, Young KH. Checkpoint inhibitors in hematological malignancies. $J$ Hematol Oncol. (2017) 10:103. doi: 10.1186/s13045-017-0474-3

11. Szalat R, Munshi NC. Novel agents in multiple myeloma. Cancer J. (2019) 25:45-53. doi: 10.1097/PPO.0000000000000355

12. van de Donk N, Richardson PG, Malavasi F. CD38 antibodies in multiple myeloma: back to the future. Blood. (2018) 131:1329. doi: 10.1182/blood-2017-06-740944

13. Moreno L, Perez C, Zabaleta A, Manrique I, Alignani D, Ajona D, et al. The mechanism of action of the anti-CD38 monoclonal antibody isatuximab in multiple myeloma. Clin Cancer Res. (2019) 25:317687. doi: 10.1158/1078-0432.CCR-18-1597

14. Ochoa MC, Perez-Ruiz E, Minute L, Onate C, Perez G, Rodriguez I, et al. Daratumumab in combination with urelumab to potentiate antimyeloma activity in lymphocyte-deficient mice reconstituted with humanNK cells. Oncoimmunology. (2019) 8:1599636. doi: 10.1080/2162402X.2019.15 99636

15. Claus C, Ferrara C, Xu W, Sam J, Lang S, Uhlenbrock F, et al. Tumor-targeted 4-1BB agonists for combination with $\mathrm{T}$ cell bispecific antibodies as off-the-shelf therapy. Sci Transl Med. (2019) 11:eaav5989. doi: 10.1126/scitranslmed.aav5989

16. Sanmamed MF, Etxeberria I, Otano I, Melero I. Twists and turns to translating 4-1BB cancer immunotherapy. Sci Transl Med. (2019) 11:eaax4738. doi: 10.1126/scitranslmed.aax4738

17. Gauthier L, Morel A, Anceriz N, Rossi B, Blanchard-Alvarez A, Grondin G, et al. Multifunctional natural killer cell engagers targeting NKp46 trigger protective tumor immunity. Cell. (2019) 177:1701-13 e16. doi: 10.1016/j.cell.2019.04.041

18. Rasche L, Chavan SS, Stephens OW, Patel PH, Tytarenko R, Ashby C, et al. Spatial genomic heterogeneity in multiple myeloma revealed by multi-region sequencing. Nat Commun. (2017) 8:268. doi: 10.1038/s41467-017-00296-y

Conflict of Interest Statement: NG received research funding and honoraria from Amgen, Bristol Mayers Squibb, Celgene, Millenium Pharmaceutical, and Janssen Pharmaceutical. FM has received honoraria for lectures and participation on the advisory boards of Janssen, Tusk Therapeutics, Takeda, and Sanofi, along with research agreements from Janssen and Tusk Therapeutics. The authors have no other relevant affiliations or financial involvement with any organization or entity with a financial interest in or financial conflict with the subject matter or materials discussed in the manuscript apart from those disclosed.

Copyright $\odot 2019$ Giuliani and Malavasi. This is an open-access article distributed under the terms of the Creative Commons Attribution License (CC BY). The use, distribution or reproduction in other forums is permitted, provided the original author(s) and the copyright owner(s) are credited and that the original publication in this journal is cited, in accordance with accepted academic practice. No use, distribution or reproduction is permitted which does not comply with these terms. 